\title{
Effect of the Performance of the Liaison Nurses on the Motor Complications in Stroke Patients after Discharge: A Randomized Clinical Trial
}

\author{
Sedighe Ebrahimi ${ }^{1}$, Zohre Kalani $^{2}$
}

\begin{abstract}
Background \& Aims: Worldwide, cerebrovascular accidents (stroke) are the second leading cause of death and the third leading cause of long-term and serious disabilities in adults. Motor and sensory impairments due to stroke cause common complications such as bedsores, constipation, and falls. In addition, strokes, directly and indirectly, affect the outcomes of patient treatment, including the duration of stay and death. Despite the rapid prevention and diagnosis and management of stroke, patients are still at high risk for complications. Given the prevalence of stroke complications, comprehensive nursing care during four weeks after a stroker plays a significant role in the decrease of the outcomes, disabilities, and mortality caused by the disorder. One of the most important roles of a liaison nurse is improving care quality by better preparing the patient for discharge. In fact, liaison nurse nurses are those who take care of patients with strokes after a holistic assessment and the application of technical and communication skills and improve their condition for discharge and continued care. Through coordination with other healthcare team members, they ensure that all the needs of patients and their families are met. In other words, the role of the liaison nurse is to improve pre-discharge planning, establish and improve communication between hospital staff and health care providers in the community, and provide home care facilities to assist hospital staff. Therefore, this study aimed to determine the effect of the performance of liaison nurses on the motor complications caused by a stroke after discharge.

Materials \& Methods: This quasi-experimental research had a randomized trial design and was performed in a hospital in Shiraz, Iran in 2016-2017. In total, 80 patients were selected by purposeful convivence sampling and were randomly divided into two test $(n=35)$ and control $(n=45)$ groups. Patients in the test group were evaluated before discharge by the liaison nurse and care and educational program focusing on the needs of each patient was developed. During discharge, an educational booklet was given to the patients or their caretakers in the test group to prevent motor complications caused by stroke (constipation, falls, bed sores). If necessary, the liaison nurse would be present at the patient's bedside at home and would be in contact with the patients to resolve their problems. On the other hand, the subjects in the control group received routine ward care and were discharged. In this study, falls were determined based on the report of patients and their families two weeks and two months after discharge (any type of fall due to imbalance and physical weakness). In addition, bedsores were determined based on the diagnosis of the liaison nurse after examining the patients, and constipation was determined by the same expert using Rome 3 criteria. Data analysis was performed in SPSS version 16 using independent t-test (to compare the mean age and criteria for stroker) and Chi-square (to compare qualitative variables). Moreover, Mann-Whitney $\mathrm{U}$ was used to compare the risk of bedsores and falls due to non-compliance with normal distribution, and the Chi-square test was used to compare the frequency of complications in the two groups.

Results: In this study, no significant difference was observed between the two groups in terms of age $(\mathrm{P}=0.319)$. the two groups were homogenous in terms of other baseline conditions, including the distribution of patients in terms of gender, Braden criterion, Morse criterion, and NIHSS. According to the results, no significant difference was observed between the groups regarding the frequency of falls two weeks $(\mathrm{P}=0.060)$ and two months $(\mathrm{P}=0.509)$ after discharge. Overall, a significant difference was observed between the two groups $(\mathrm{P}=0.041)$. Moreover, the groups had no significant difference regarding the frequency of constipation two weeks $(\mathrm{P}=0.121)$ and two months $(\mathrm{P}=0.102)$ after discharge, but the overall difference in the frequency of constipation was significant $(\mathrm{P}<0.001)$. In terms of bedsores, no significant difference was observed between the two groups two weeks $(\mathrm{P}=0.861)$ and two months $(\mathrm{P}=0.037)$ after discharge and in total $(\mathrm{P}=0.068)$.
\end{abstract}

\footnotetext{
1. MS in Critical Care Nursing, School of Nursing and Midwifery, Shahid Sadoughi University of Medical Sciences, Yazd, Iran

2. Instructor, Nursing and Midwifery Care Research Center, Shahid Sadoughi University of Medical Sciences, Yazd, Iran (Corresponding author)

Tel: $09133524935 \quad$ Email: z.kalani@ @su.ac.ir
} 
Conclusion: In this study, no significant difference was observed in the frequency of bed sores following the intervention of a liaison nurse. One of the positive points in this study was the use of the Braden criterion to predict the risk of bedsores and plan care based on the results in the test group. The criterion evaluates sensory perception, immobility, incontinence, and nutritional status. For each of the factors, separate measures were considered by the liaison nurse in order to reduce the occurrence of further problems and patient referral due to high-grade and infectious bed sores. According to the results of the study, the intervention of the liaison nurse significantly decreased the frequency fall in the test group, compared to the control group. By assessing the risk of falls in stroke patients based on Morse criterion, the liaison nurse increased sensitivity toward the topic in the patients and their families and pointed out the causes of falls. In the control group, two patients fell despite the low risk of falling according to Morse's criterion, which might be due to the insufficient knowledge of patients and their caregivers about the issue in the absence of a liaison nurse. The results were different in terms of the incidence of falls with the clinical trial of the fall prevention program with several factors that were performed at home after the rehabilitation phase of stroke patients. We found a significantly lower frequency of constipation in the test group. One of the expected results in this trial was the implementation of self-care behaviors in patients with stroke. Since sampling was carried out in a short duration and only in two internal brain and nervous systems and brain ICU sectors, it seems that the implementation of the liaison nurse plan on stroke patients to achieve more accurate results requires more time and a larger sample size. Some of the important measures to be taken by liaison nurses include special care and education of these individuals and following up patients after discharge. According to the results, the performance of liaison nurses regarding stroke patients was effective in the decrease of motor complications such as constipation and falls. However, they played no role in the decrease of bedsores in these patients.

Keywords: Stroke, Liaison Nurse, Bedsores, Constipation, Fall

\section{Conflict of Interest: No}

How to Cite: Ebrahimi S, Kalani Z. Effect of the Performance of the Liaison Nurses on the Motor Complications in Stroke Patients after Discharge: A Randomized Clinical Trial. Iran Journal of Nursing. 2019; 32(120):14-25.

Received: 6 Jul 2019

Accepted: 7 Oct 2019 


\title{
تأثير عملكرد يرستار رابط بر فراوانى عوارض حركتى ناشى از سكته مغزى بس از ترخيص از بيمار ستان: يك كار آزمايى بالينى تصادفى
}

\author{
صديقه ابراهيمى '، زهره كلانى
}

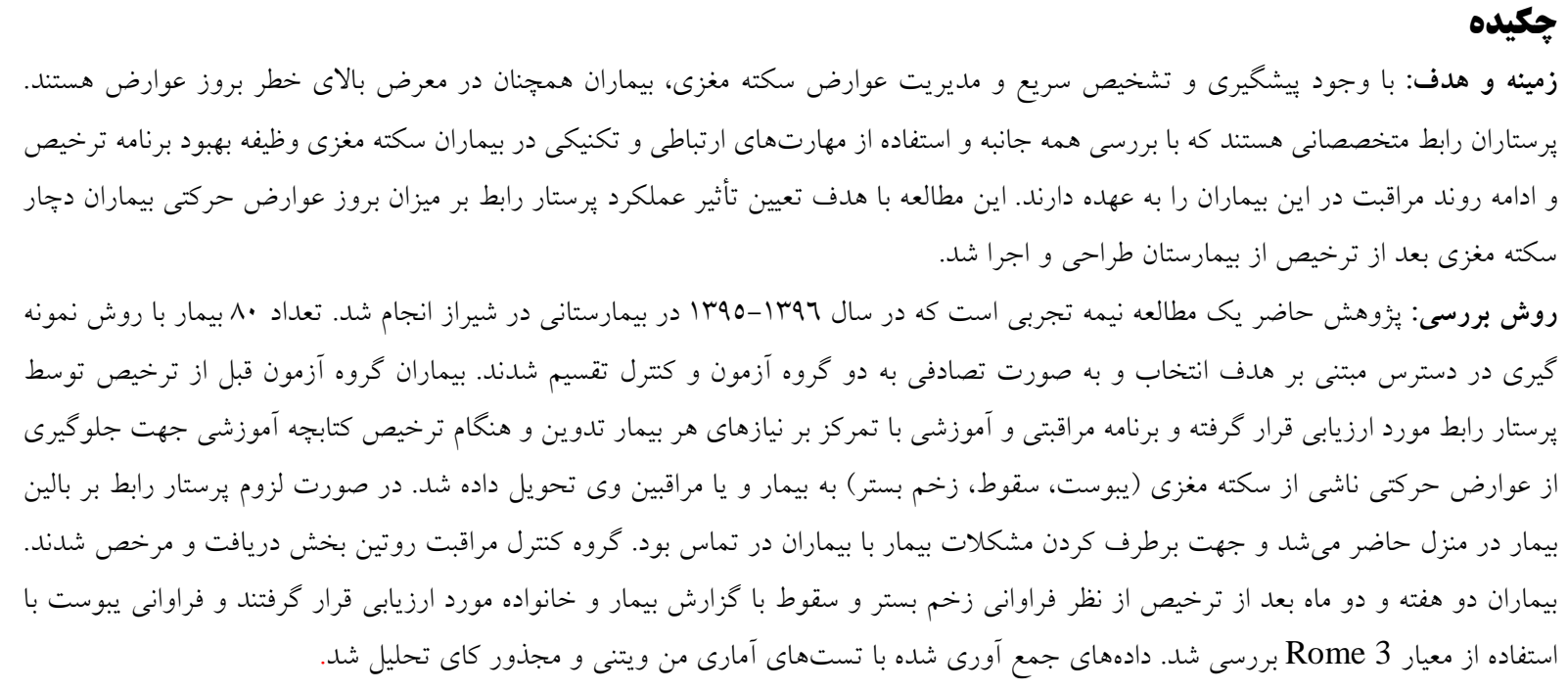

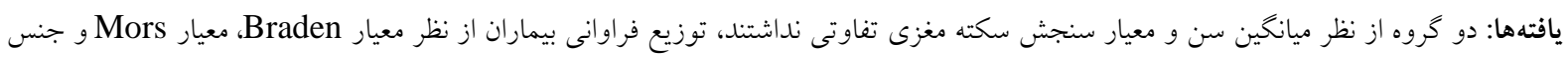

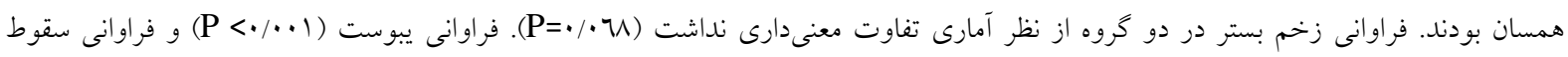

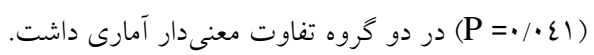

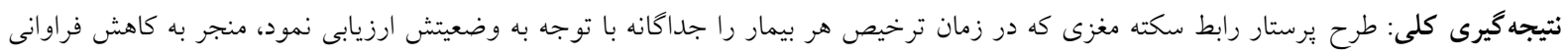

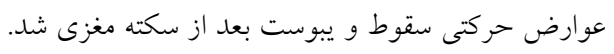

كليد وازهها: سكته مغزى، يرستار رابط، زخم بستر، يبوست، سقوط تعارض منافع: ندارد تاريخ دريافت: تاريخ يذيرش:

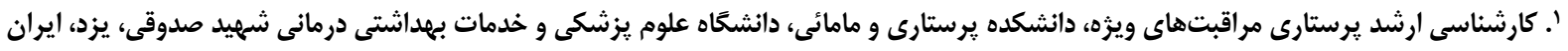

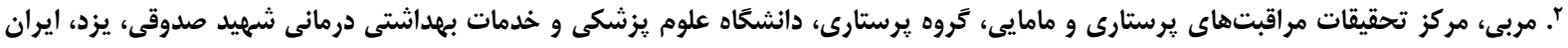
Email: z.kalani@ssu.ac.ir 


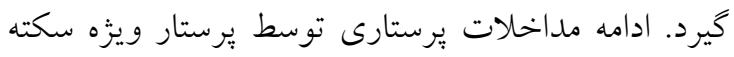

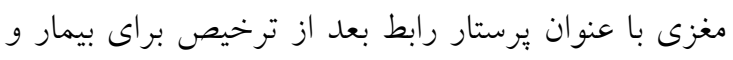

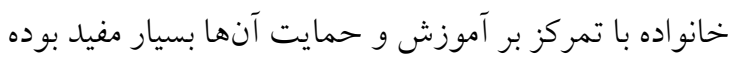

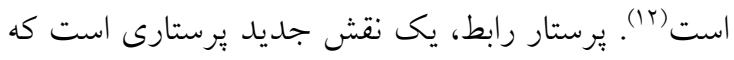

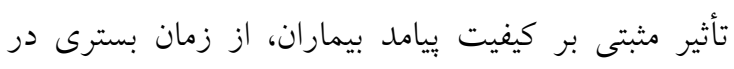

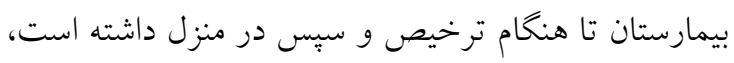
به ويزه در بيمارىهاى مزمن، مىتواند از بسترى مجدد بيمار جلو گيرى و ادامه مراقبتها را بهبود بخشد. امروزه بسيارى از خدمات ارئه دهنده سلامت در دنيا بر نقش يرستار رابط يا برستار هماهنگ كننده ترخيص تمركز كرده اند. يرستار رابط با هماهنگى با ساير اعضاى تيم درمان اطمينان حاصل مى كند كه تمامى نيازهاى بيمار و خانواده

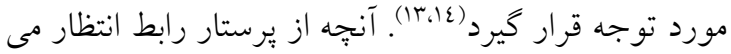
رود داشتن علم و مهارت كافى جهت ادامه مراقبت از بيمار و ارتباط با ساير يرستاران و در نهايت بهبود بيامدهاى بيمار

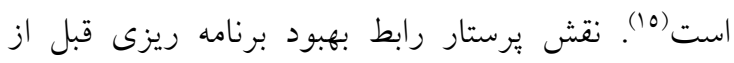

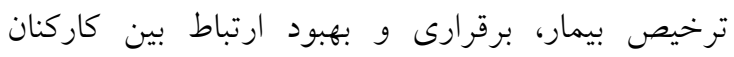

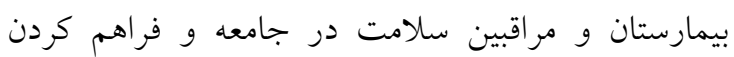
امكانات مراقبت در منزل جهت كمك به كاركنان بيمارستان

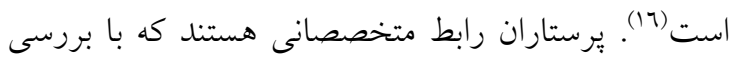

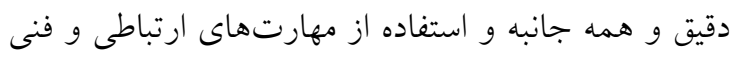
به مراقبت از بيماران داراى مشكلات متعدد نظير بيماران

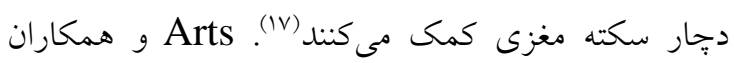

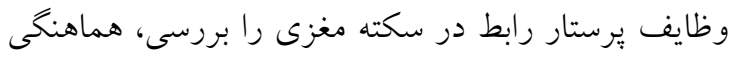

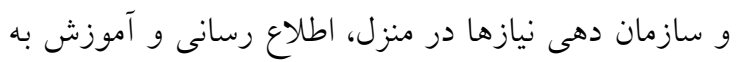
مراقبت دهنده بيمار، سريرستى ارتباط بين بيمارستان و ادامه مراقبت در منزل، ارزيابى و تشخيص خطا در روند مراقبتها بيان مىكنند و نقش يرستار رابط را در زمان ترخيص بيمار و انتقال به منزل، بسيار مؤثر يافتند (11).

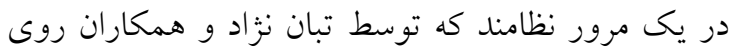
بررسى نقش يرستار رابط در يِيامدهاى بيمار بعد از ترخيص از بخش مراقبتهاى ويزّه انجام گرفت، جهار مطالعه مرور شده، تأثير رضايت بخش مشخصى را در

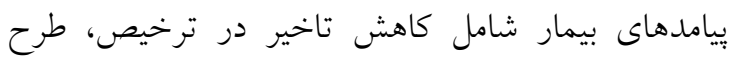

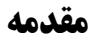

سكته مغزى دومين عامل مرى در جهان و سومين عامل شايع ناتوانى هاى بلندمدت و جدى در بزركسالان است (1) و به عنوان يك مشكل مهم بهلاشتى در خاورميانه، تخمين زده مىشود كه مرگ ناشى از آن تا سال •r.r.r به دو برابر

برسد (r) (ب). دو سوم از بيماران بعد از سكته مغزى حداقل يكى عارضه و در طى سه ماه بعد از سكته مغزى جهار تا ينج عارضه را تجربه مى كنند (r). عوارض متعاقب سكته مغزى هم به طور مستقيم و هم غيرمستقيم بر بيامدهاى درمان در اين بيماران شامل طول مدت اقامت و مرگ مؤثر هستند(ع). اختلال حركتى و حسى ناشى از سكته مغزى منجر به عوارض شايعى مثل زخم بستر، يبوست و سقوط مى شود (0). در بيمار ان مبتلا به سكته مغزى به علت بى حركتى طولانى مدت بروز زخم فشارى شايع است. زخم بستر علاوه بر درد و رنج بسيار، مىتواند منجر به ناتوانى و مرگ شود. بيشخيرى مؤثرترين روش براى حل

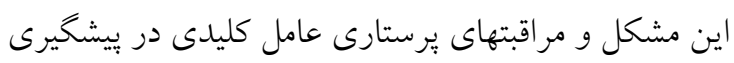
است(7). در مطالعه مشكات حركات رودهاى در فاز بازتوانى در بيماران سكته مغزى بيان شده است كه .0

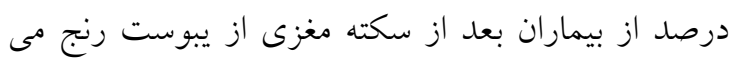

برند (v) در مطالعه Ashburn و همكاران فراوانى سقوط در 00 درصد از بيماران دجار سكته مغزى يك تا دو بار طى ماه بعد از سكته مغزى وجود داشته است (^). با توجه به شيوع عوارض در بيمار ان با سكته مغزى، مراقبت هاى يرستارى جامع در طول مدت جهار هفته بعد از سكته در كاهش عوارض، ناتوانى و مرگ بسيار مؤثر است (9). شكاف بين بيمارستان و سيستمهاى حمايتى از بيمار در جامعه منجر به افت كيفى مراقبتها و ادامه درمان مى شود (·) . بهبود كيفيت مراقبتها با آماده سازى بهتر بيمار جهت ترخيص، يكى از اهداف مهم برستار رابط است (11). فرايند ترخيص بيمار و انتقال وى به منزل براى بيمار و

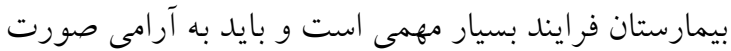


در ايران انجام شده است. اين مطالعه با هدف تعيين تأثير عملكرد يرستار رابط بر عوارض حركتى بيماران دجار

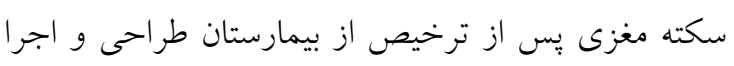
كرديد.

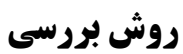

اين يزّوهش يكى مطالعه نيمه تجربى بالي طرح كارآزمايى

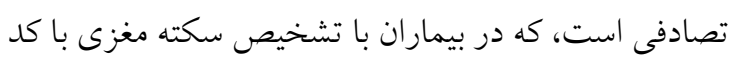

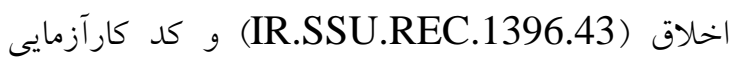
بالينى (IRCT20170605034330N3)  قبل از بسترى در بيمارستان حضرت على اصغر شيراز در

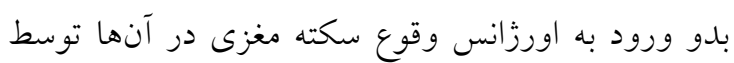
متخصص مغز و اعصاب با انجام سى تى اسكن تأييد شده ونه

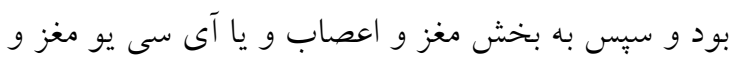
اعصاب منتقل شده بودند.
ترخيص مؤثر، كاهش بسترى مجدد بيمار را نشان دادند.

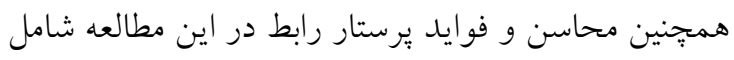

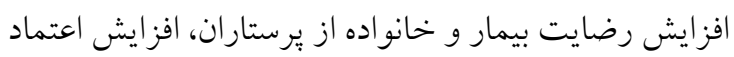

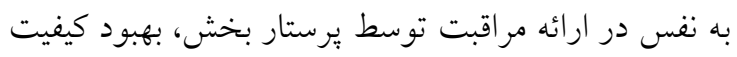

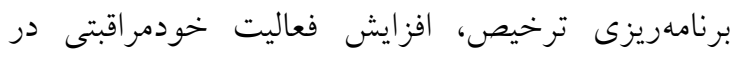
بيمار انو كاهش تعداد روزهاى بسترى در بيمارستان بود (1).

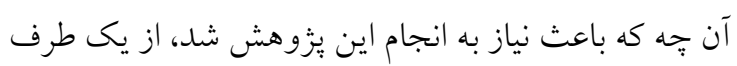

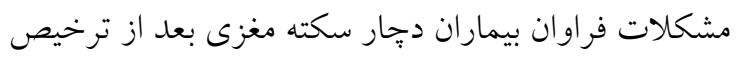
و ضرورت ادامه مراقبت در منزل و نبود سازمان حمايت

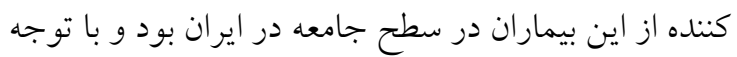

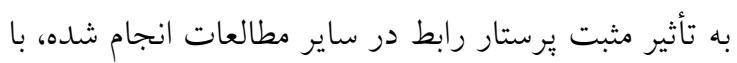

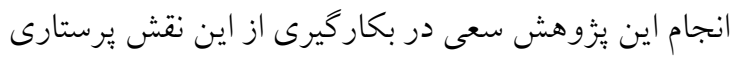
در برطرف كردن مشكلات بيماران سكته مغزى شد. در سيستم مراقبتى بيماران در ايران اين نقش پِرستار كاملاً نا آشنا است و تاكنون يزوهشهاى محدودى در ارتباط با يال

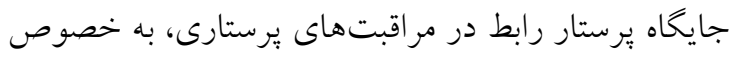

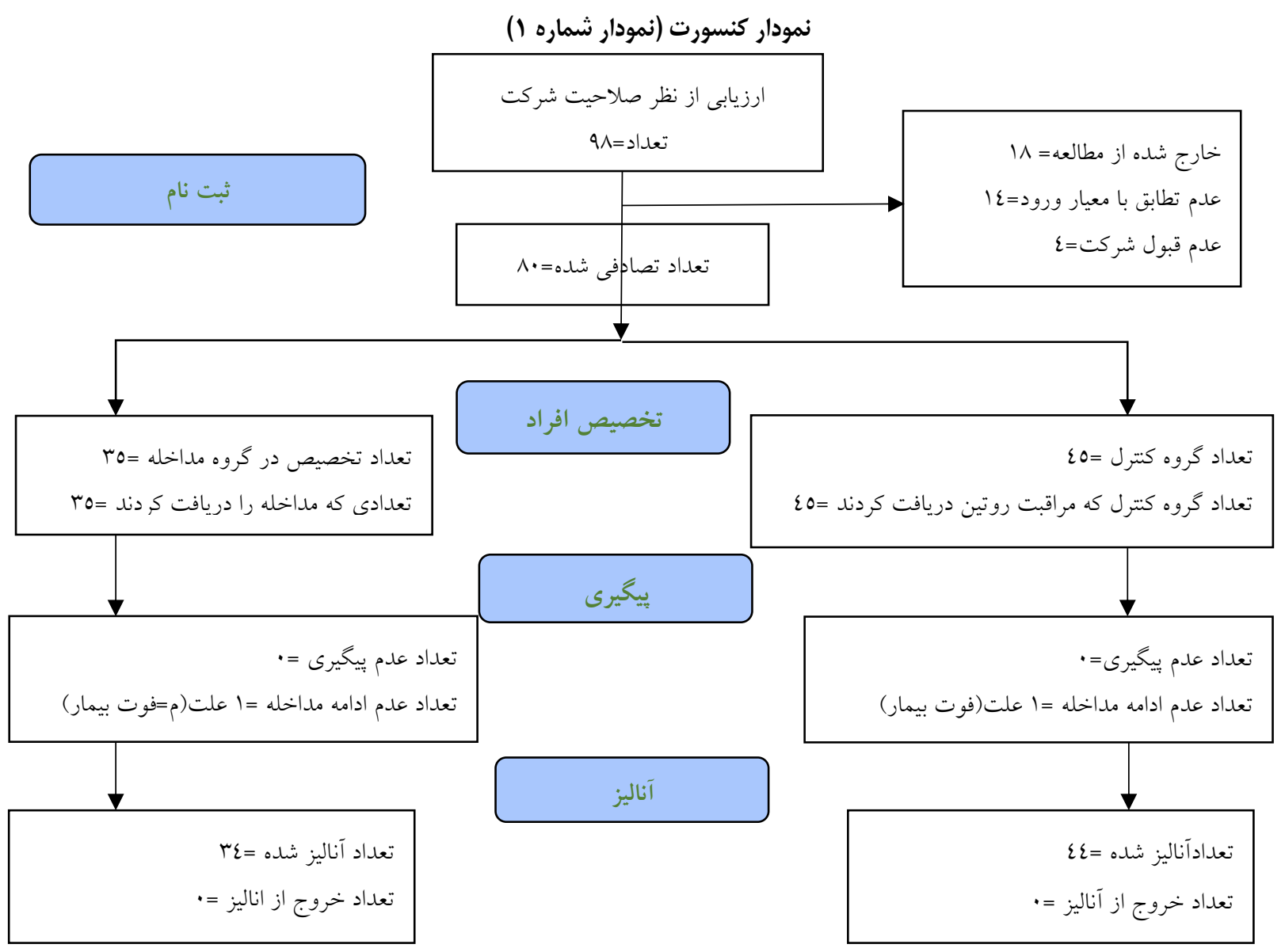


Braden سايش، تحرى، جابجايى، ميزان تغذيه سنجيده مىشود و

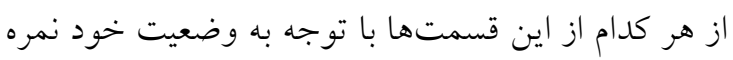

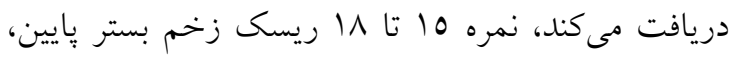

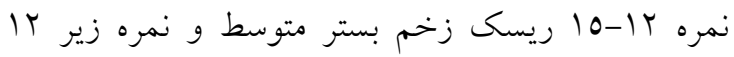

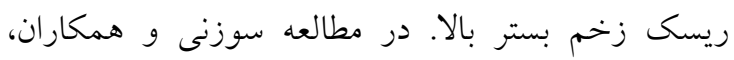

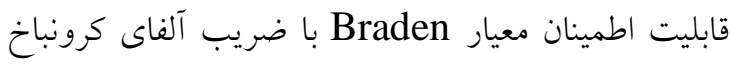

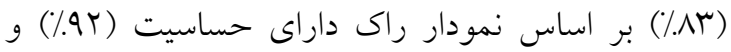

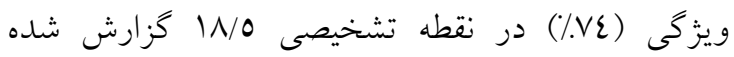

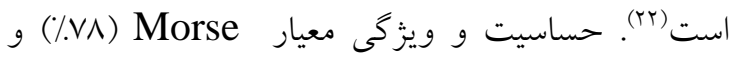

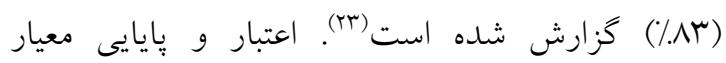
Morse توسط شاهى و همكاران تاييد شده است (ع) . در اين معيار ريسك فاكتورهاى مستعد كننده بيمار جهت بروز سقوط شامل سقوط طى سه ماه كذشته، وضعيت مغزى، استفاده

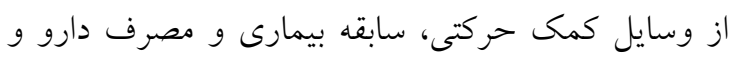

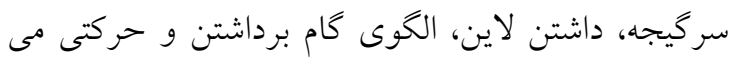

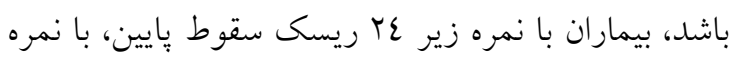

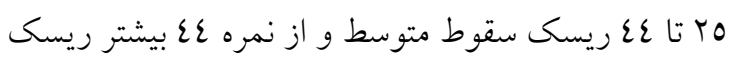
سقوط بالا دارند. خطر بروز يبوست با استفاده از معيار Rome3

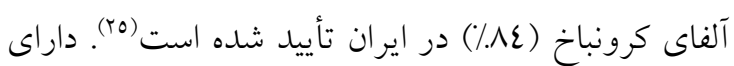

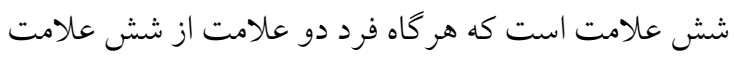

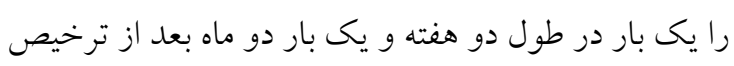

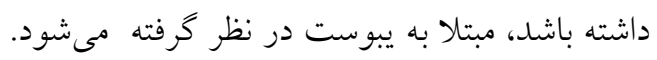

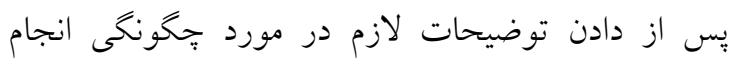

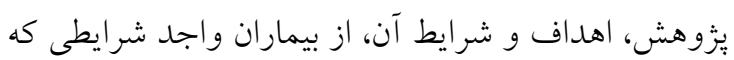
تمايل به شركت در مطالعه داشتند، رضايت نامه كتبى اخذ كرديد. در اين مطالعه يزوهشخر به عنوان يرستار رابط براى

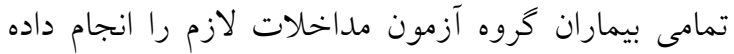
است. يرستار رابط در اولين روز بسترى در گروه آزمون بـان

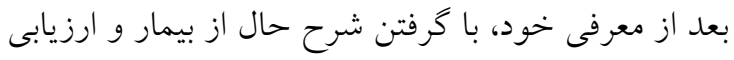

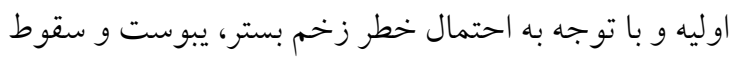

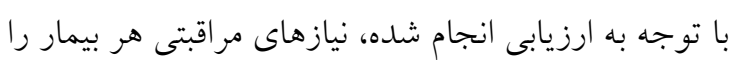

حجم نمونه با استفاده از فرمول محاسبه حجم نمونه براى

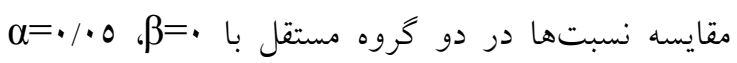

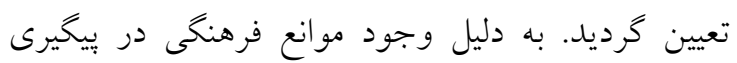
بيماران در منزل توسط يرستار رابط با استفاده از نسبت ورديت

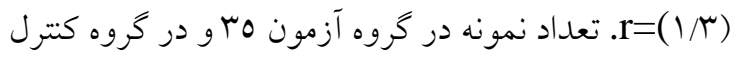
0ح بيمار در نظركرفته شد. بيماران با توجه به معيارهاى

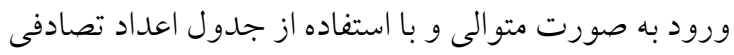
در خروه هاى آزمون و كتترل قرار گرفتند (نمودار شماره

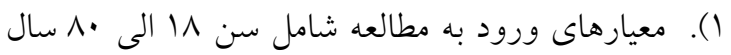
و اخذ نمره 17 الى ·r بر اساس معيار سنجش سكته مغزى (National Institutes of Health Stroke Scale, بود. روايى اين ابزار توسط بسامى و همكاران NIHSS)

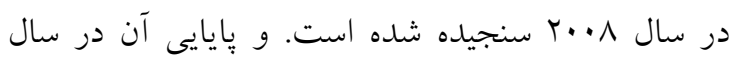

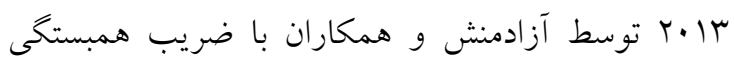

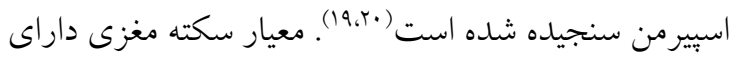

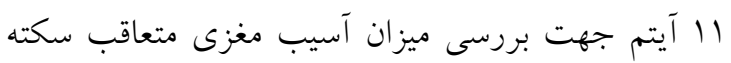

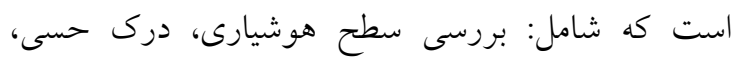
بررسى تعادل، صحبت كردن، رفلكس قرنيه، اختلال تكلم،

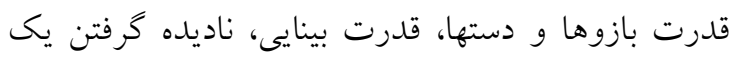

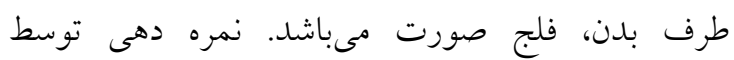

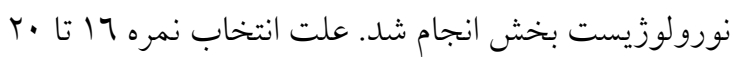
وضعيت بيمار جهت انجام مداخله بوده است، بيماران با لتان

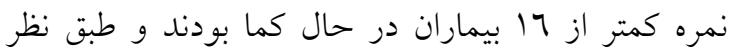
نورولوزيست بيش آَّهى خوبى نداشتند و بيماران با نمره بيشتر از • ب قادر به مراقبت كامل از خود هستند و نياز به مداخله يرستارى در منزل نداشتند. نسخه فارسى اين ابزار

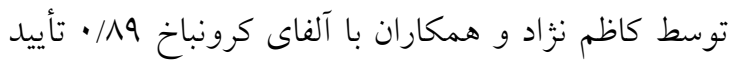

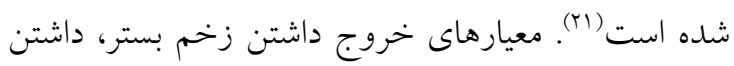
يبوست، داشتن بى حركتى قبل از سكته بودند.

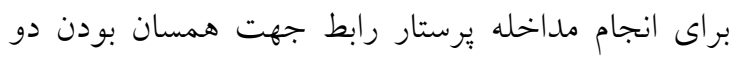

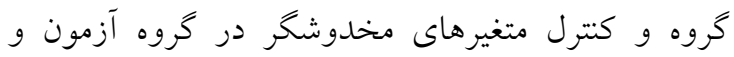

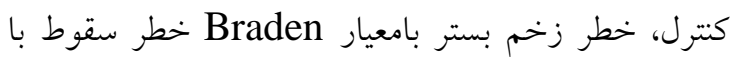

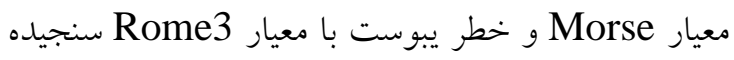
شدند كه از نظر آمارى تفاوت معنى دارى نداثتند. در معيار 
و آموزشص مشى هرد و با توجه به اولويت هر بيمار مراقبتها

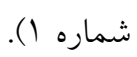

جدول شماره (: مداخلات يرستار رابط در ارتباط با احتمال بروز هر يك از عوارض حركتى ناشى از سكته مغزى

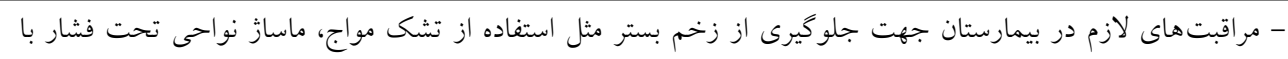

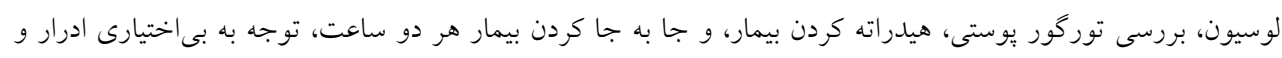

$$
\text { مدفوع و انجام مراقبت. }
$$

- بررسى نيازهاى آموزشى در منزل و آموزش خانواده در اين زمينه.

- بررسى وضعيت تغذيه و تنظيم برنامه غذايى مناسب جهت حفظ تماميت يوستى با هماهنكى كارشناس تغذيه.

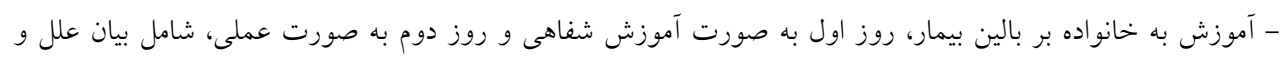

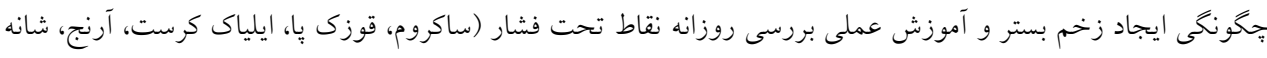

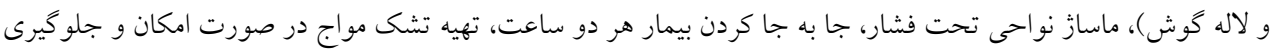
از خيس بودن و خروكيدگى ملحفهها. ويزيت در منزل توسط برستار رابط در هنكام تماس بيمار يا خانواده مبنى بر زخخم بستر

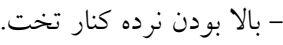
- مدر دسترس بودن وسايل مورد نياز. - استفاده از وسايل كمك حركتى حين حركت كردن.

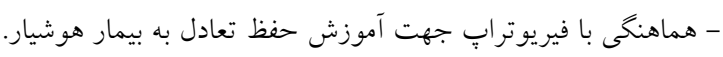

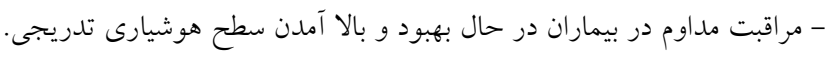

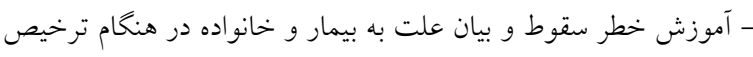

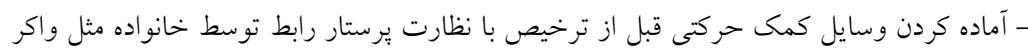

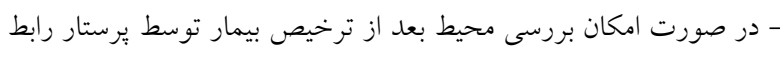

- آموزش مصرف مايعات، سبزيجات در بيمار هوشيار

- خروج هرجه سريعتر از تخت در بيماران هوشيار.

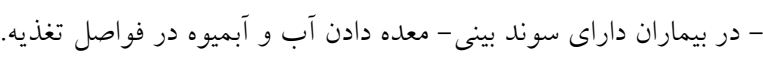

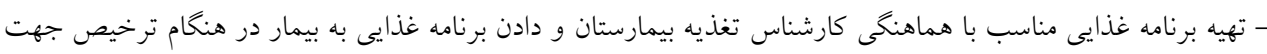
جلو كيرى از يبوست در منزل - آموزش نشا نههاى يبوست به بيمار و خانواده

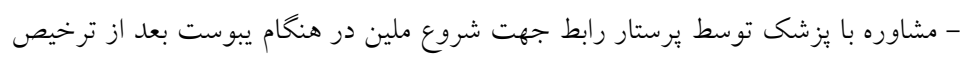

(يكى از اعضاء خانواده كه در تماس بيشترى با بيمار بود

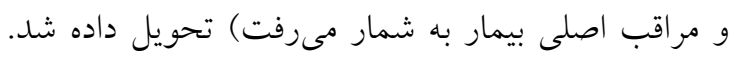
شماره تلفنى جهت تماس با يرستار رابط به بيمار و خانو اده داده شد. در طول دو ماه بعد از ترخيص در صورت نياز،
طى دوره بسترى آموزشها به صورت عملى و شفاهى

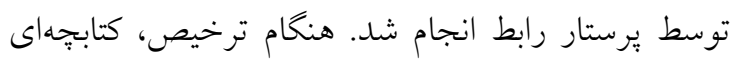

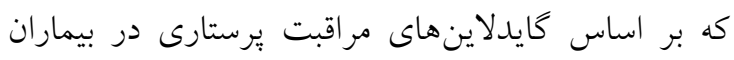

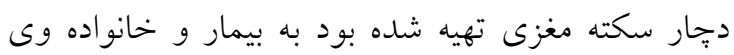


ج) ليست بروز عوارض يكى باردو هفته و يك بار دو ماه بعد از ترخيص تكميل كرديد.

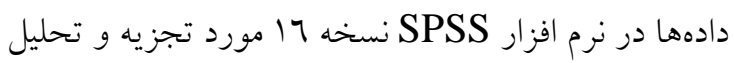

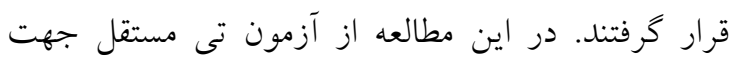

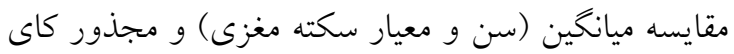
جهت مقايسه متغيرهاى كيفى استفاده شد. جهت مقايسه خطر زخم بستر و سقوط به دليل عدم تبعيت از توزيع نرمال، از آزمون من ويتنى و همجنين جهت مقايسه فراوانى

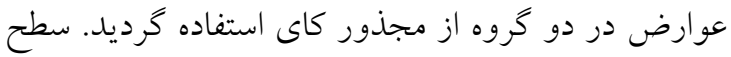
معنى دارى در اين مطالعه 0٪/ در نظر كرفته شد.

يافتهها دو گروه مورد بررسى از نظر سنى تفاوت معنىدارى

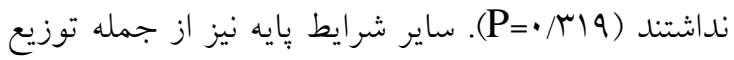
بيماران از نظر جنسيت، معيار برادن، معيار مورس و NIHSS

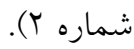

بيمار در منزل توسط يرستار رابط ويزيت شده و مشكلات

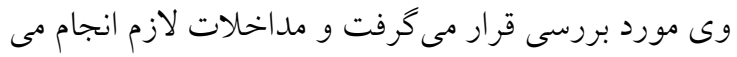

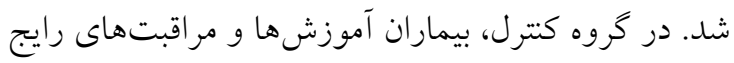
بخش را دريافت نمودند. يك بار دو هفته و يك بار دو ماه بعد از ترخيص، همزمان با ويزيت بيمار توسط يزشك، بيماران از نظر فراوانى زخم

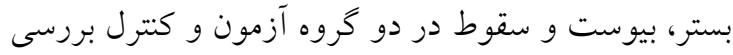

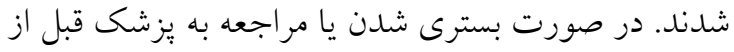
دو هفته به هر دليل، به يرستار رابط اطلاع داده مى شـد.

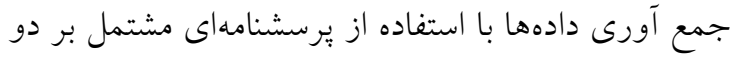
بخش صورت كرفت. بخش اول خصوصيات جمعيت شناختى بيماران و بخش دوم جیى ليست فراوانى عوارض بر اساس اهداف يزوهش بود.

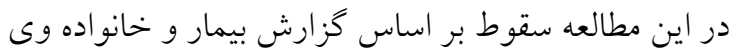

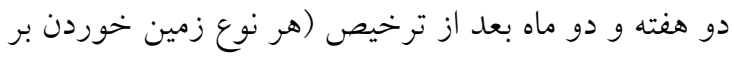

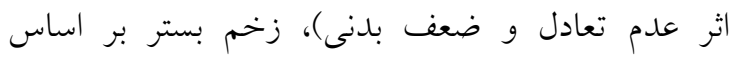

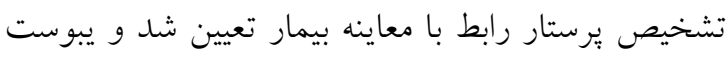

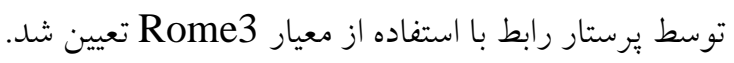

جدول شماره ז: مشخصات تروه كنترل و آزمون از نظر سن،جنس،ضعف بدنى،

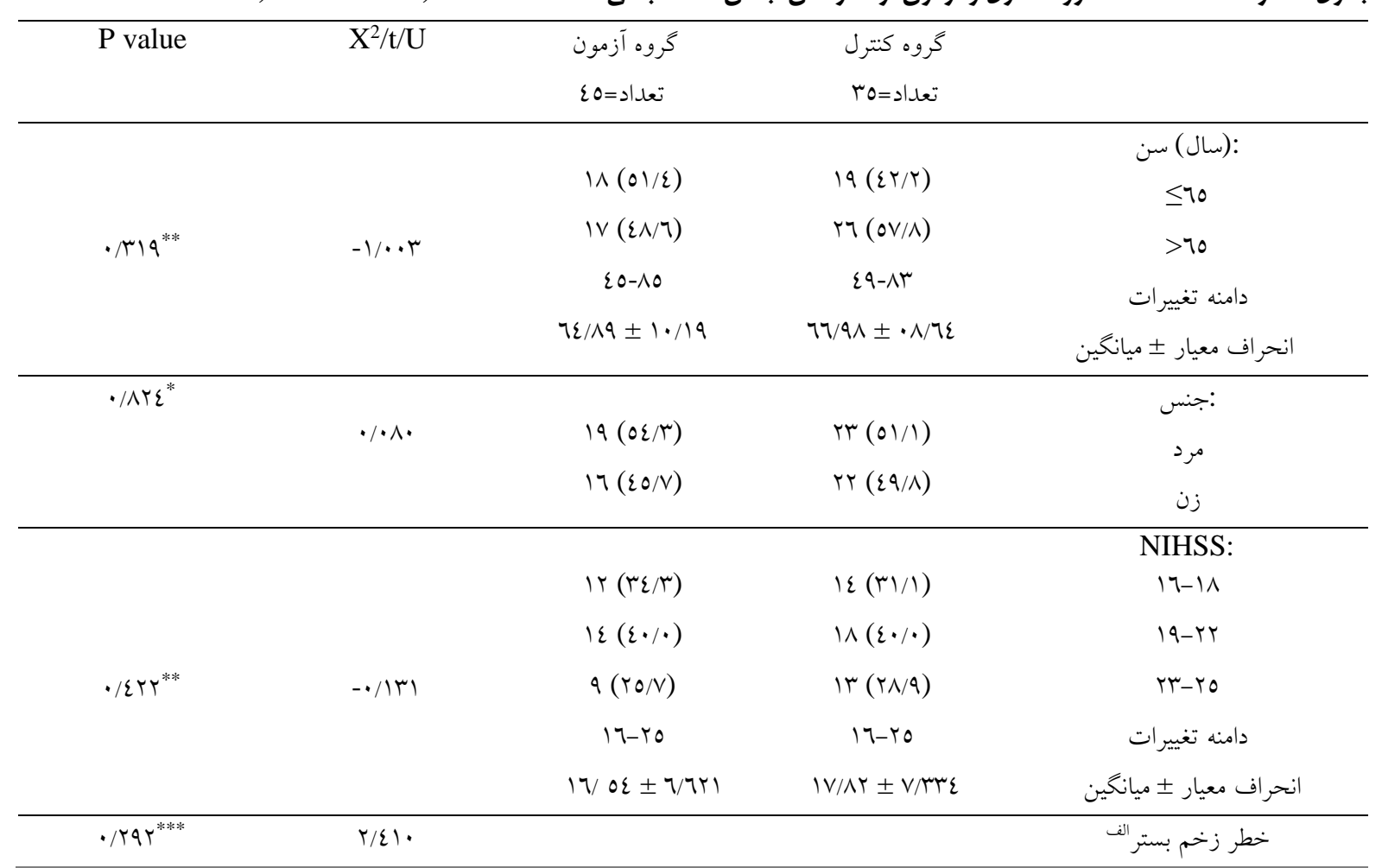




\begin{tabular}{|c|c|}
\hline $\begin{array}{l}11(r / / \varepsilon) \\
7(\mid V / 7) \\
11(0 / / \varepsilon)\end{array}$ & 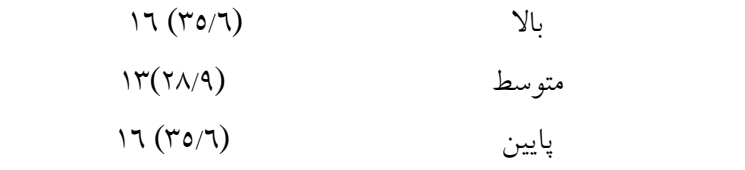 \\
\hline $\begin{array}{l}1 \wedge(01 / \varepsilon) \\
1 \cdot(T / r \Lambda) \\
\vee(r \cdot)\end{array}$ & 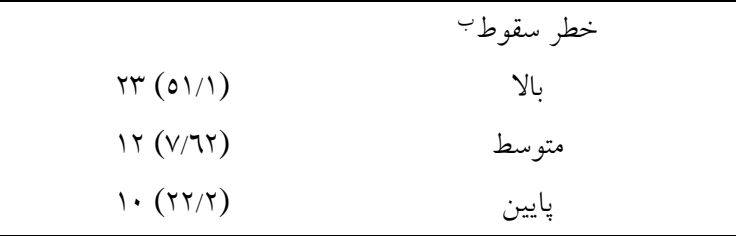 \\
\hline 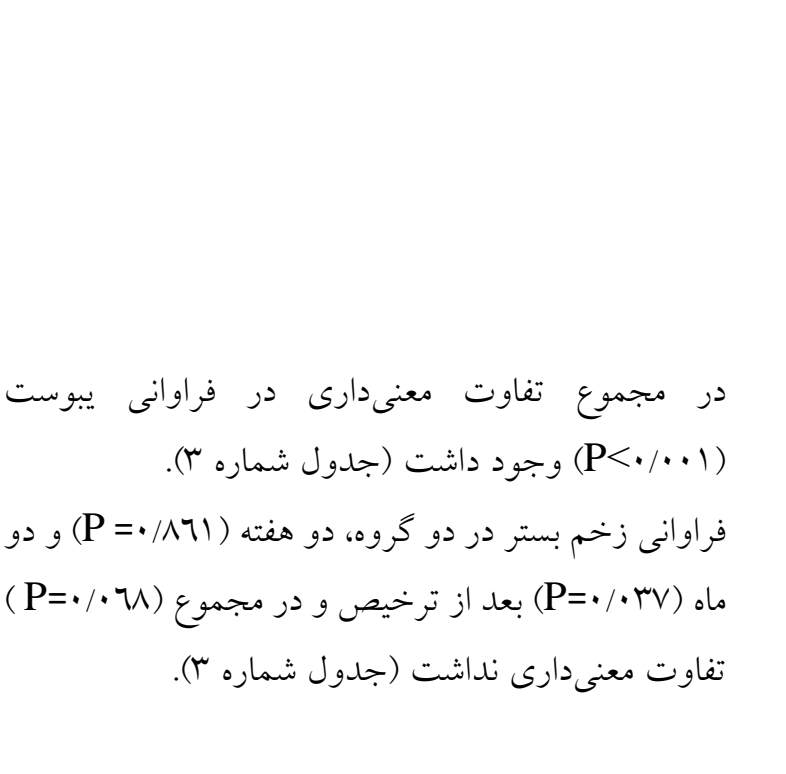 & 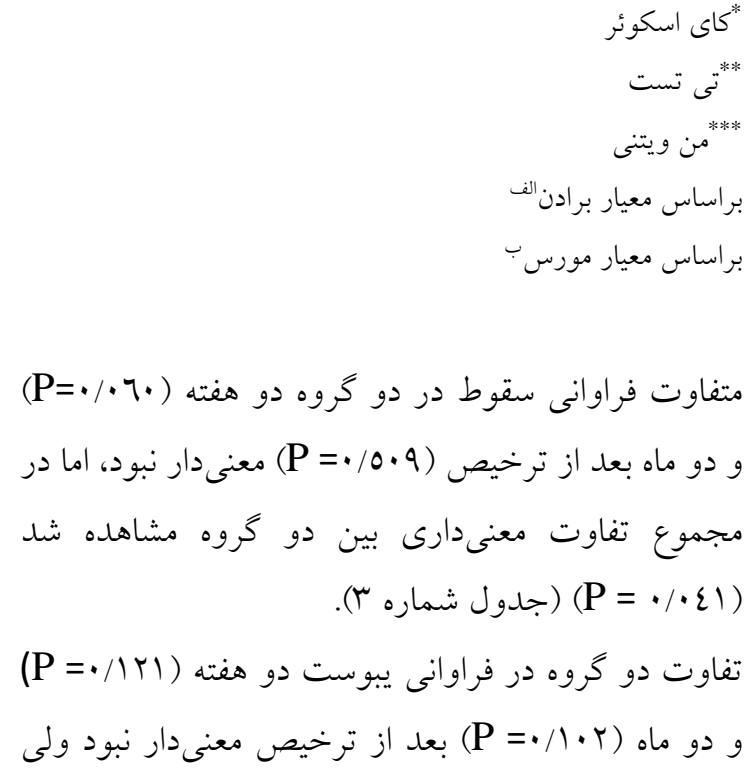 \\
\hline
\end{tabular}

جدول شماره ب: فراوانى عوارض حركتى در بيماران تروه آزمون و كنترل بعد از مداخله

\begin{tabular}{|c|c|c|c|c|c|}
\hline "P value & $X^{2}$ & كروه كتترل & كروه آزمون & & \\
\hline$\cdot / / 171$ & $\cdot / \mu \cdot$ & $r(T / V)$ & $r(O N)$ & بعد از دو هفته & \\
\hline$\cdot / \cdot r v$ & $\varepsilon / \pi 70$ & $\wedge(1 N / 9)$ & $1(r / 9)$ & بعد از دو ماه & زخم بستر \\
\hline$\cdot / \cdot \mathrm{u}$ & r/乏^. & $\|(\mathrm{rON})$ & $r(9 / N)$ & مجموع & \\
\hline .1 .7 & r/OYV & $V(10 / 7)$ & $1(T / 9)$ & بعد از دو هفته & \\
\hline $.10 \cdot 9$ & . /2ro & $7(1 \Gamma / 7)$ & $r(N / \Lambda)$ & بعد از دو ماه & سقوط \\
\hline$\cdot / \cdot \varepsilon \mid$ & $r / 01$. & $\mathbb{1 r}(r q / r)$ & $\varepsilon(11 / v)$ & مجموع & \\
\hline.$/|T|$ & $r / \varepsilon \cdot \Lambda$ & $\| r(r / / q)$ & $0(1 \xi / \Gamma)$ & بعد از دو هفته & \\
\hline$\cdot / \cdot r$ & $r / T V$. & $1 \cdot(Y Y / V)$ & $r(\Lambda / \Lambda)$ & بعد از دو ماه & يبوست \\
\hline$. / . \cdot 1<$ & $\varepsilon /$ Tor & $r \mu(01 / V)$ & $\wedge(\Gamma \mu / 1)$ & مجموع & \\
\hline
\end{tabular}

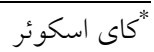

به حداقل رســاندن ابتلا به زخم بسـتر مبحث مهمى در مراقبت از بيماران با شرايط بحرانى ا ست. در يكى مطالعه

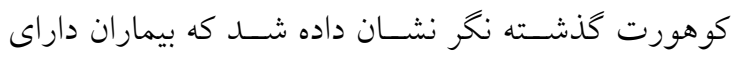
زخم بسـتر به طور معنى دارى در معرض خطر بالاى نداى

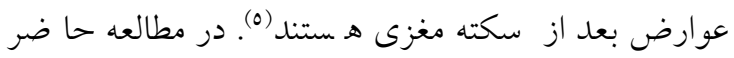

\section{بحث و نتيجه كيرى} نتايج اين مطالعه نشان داد كه عملكرد يرستار رابط منجر به به فيه كاهش فراوانى سقوط و يبوست در بيماران مبتلا به سكته مغزى مىشود ولى تأثيرى بر فراوانى زخم بستر اين بيماران 


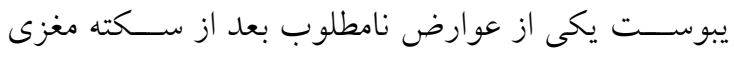
است (T9). فلج بدن و بك تحركى، داشتن سوند بينى - معدهه،

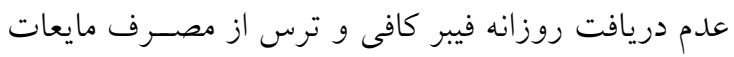

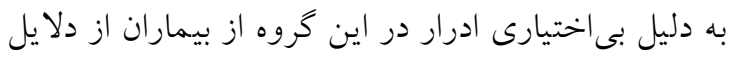

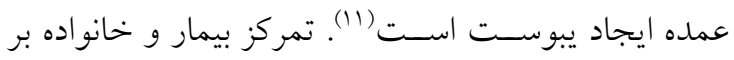
ســاير جنبه هاى بيمارى مثل بازتوانى و در اولويت قرار

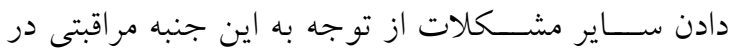
بيماران مى كاهد. توجه به وضــعيت دفعى در اين بيماران

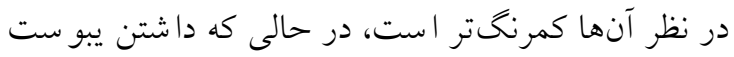
باعث عدم راحتى بيمار، بىا شتهايى، درد شكمى شده، و

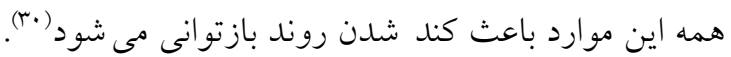
در مطالعه حاضر، فراوانى يبو ست در بيماران كروه كنترل مشـــابه مطالعه Englar وهمكاران بود و ولى در كروه تئه آزمون فراوانى يبوسـت به ميزان بسـيار كمترى ديله شـد.

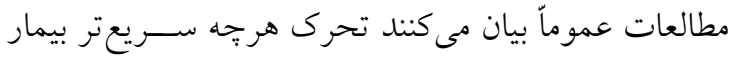

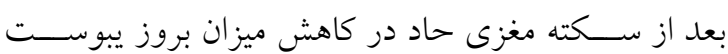

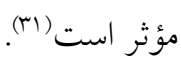
بعضى مطالعات هم بر روى اثر مداخلات يرستارى جامعه

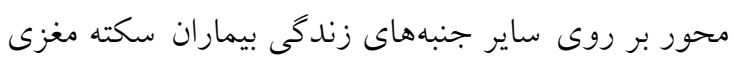

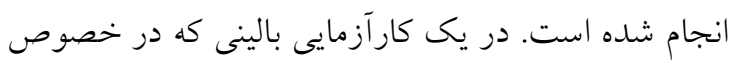

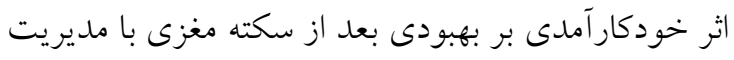

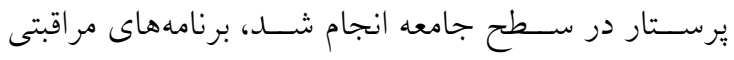
شــامل ويزيت جهار هفته بعد از ســكته مغزى در منزل توســط برسـتار و ييخيرى بيمار با تماس تلفنى با بيمار

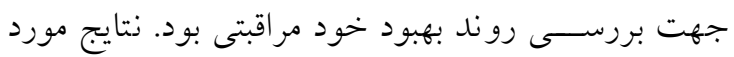

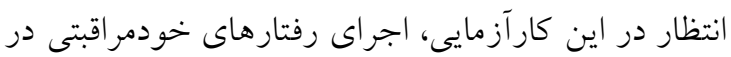

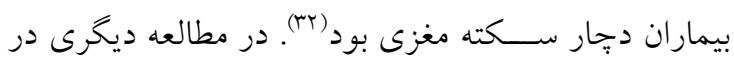

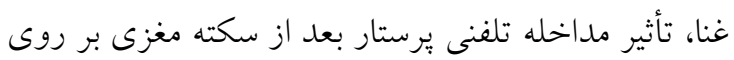
بهبود كنترل فشـار خون و تداوم مصسرف داروها يكى ماه بعد از شروع علايم در بيماران سنجيده شد (بT). در مطالعه حاضر نمونه گيرى در يكى دوره كوتاه و فقط از

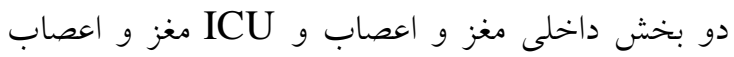

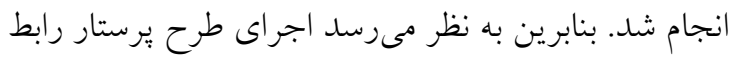

نشان داده شد كه با مداخله يرستار رابط تفاوت معنىدارى در فراوانى زخم بستر مشاهده نشد. فراوانى زخم بستر در

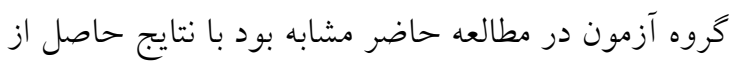

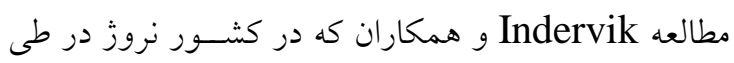

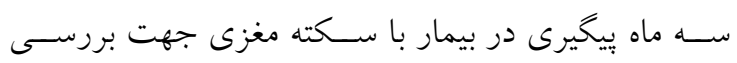

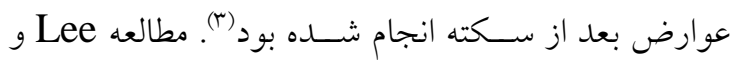

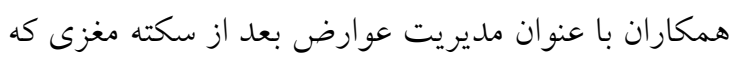

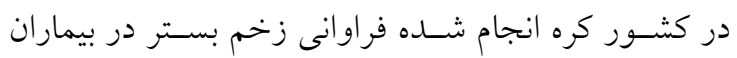

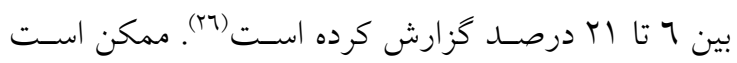

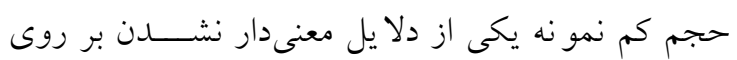

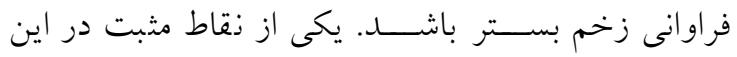

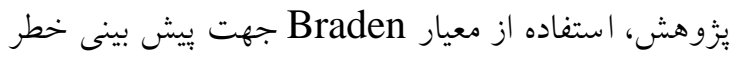

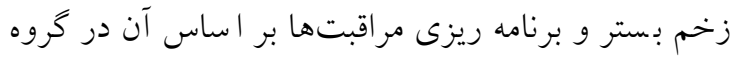

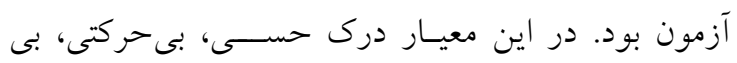

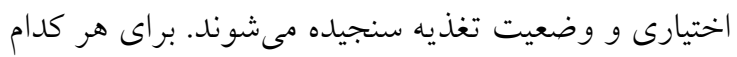

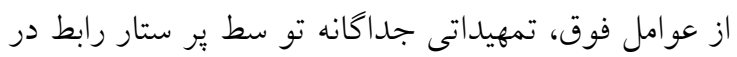

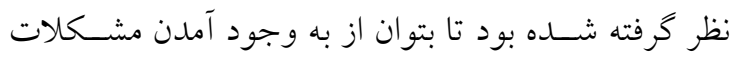

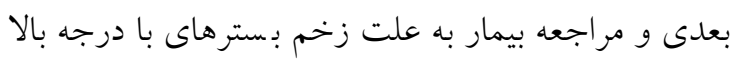
و عفونى كاست.

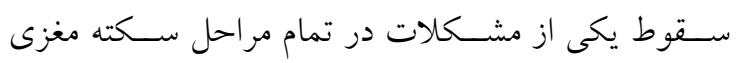

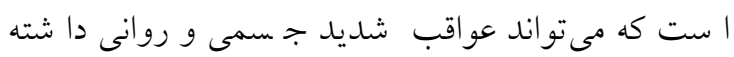

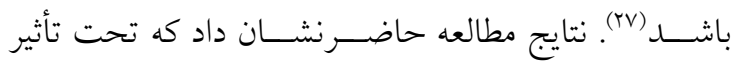

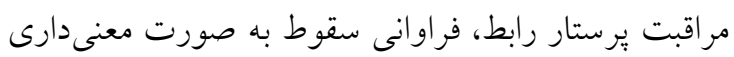

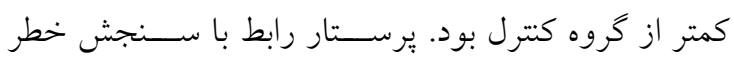

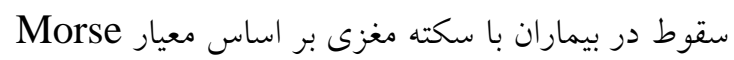

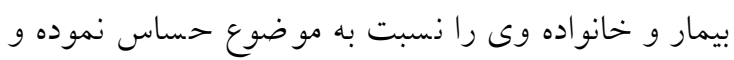

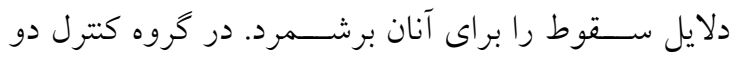

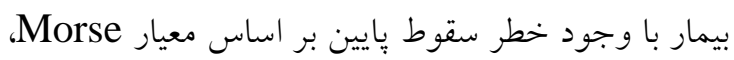

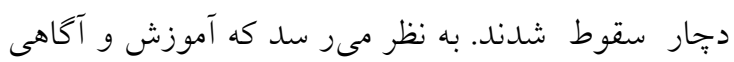
ناكافى بيماران و مراقبين در غياب يرسـتار رابط در اين

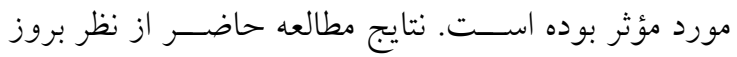

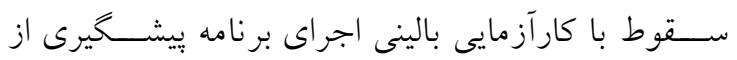

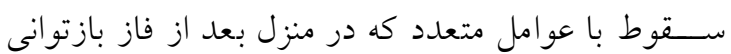
بيماران سكته مغزى انجام شد، متفاوت بود (^^). 


$$
\begin{aligned}
& \text { بهداشت و درمان از اين نقش يرستارى به خوبى استفاده } \\
& \text { بر روى بيماران سكته مغزى جهت دستيابى به نتايج دقيق } \\
& \text { تر نيازمند زمان و حجم نمونه بيشتر باشد. } \\
& \text { تعارض منافع: هيحگُنه تضاد منافع توسط نويسندگان } \\
& \text { آموزش و مراقبت ويزه يرستار رابط و از همه مهمتر رها } \\
& \text { كزارش نشده است. } \\
& \text { نشدن بيمار بعد از ترخيص و ادامه مراقبت و يايش بيمار } \\
& \text { در منزل از اقدمات مؤثر برستار رابط است. نتايج اين } \\
& \text { تقدير و تشكر } \\
& \text { مطالعه نشان داد كه عملكرد يرستار رابط در مراقبت از } \\
& \text { با تشكر از بيماران و خانوادههاى آنها كه در انجام اين } \\
& \text { يزوهش همكارى نمودند. } \\
& \text { بيماران دجّار سكته مغزى در كاهش فراوانى عوارض تر } \\
& \text { حركتى بعد از سكته مثل يبوست و سقوط مؤثر است اما بر } \\
& \text { فراوانى زخم بستر اثرى ندارد. بيشنهاد مىشود مطالعات } \\
& \text { بيشترى در زمينه استفاده از خدمات برستار رابط و به ويزه } \\
& \text { در ساير بيمارىهاى مزمن انجام شود، تا بتوان با نتايج } \\
& \text { قطعى و بيشتر در سطح كلان برنامه ريزى در سيستم }
\end{aligned}
$$

\section{References}

1. Edwardson M A, Dromerick A W, Kasner S K._Ischemic stroke prognosis in adults. www.Up To date.com/content/ischemic-stroke-pronosis-in-adults.28 Dec., 2015

2. Fahimfar N, Khalili D, Mohebi R, Azizi F, Hadaegh F. Risk factors for ischemic stroke; results from 9 years of follow-up in a population based cohort of Iran. BMC neurology. 2012;12(1):117. [Persian]

3. Indredavik B, Rohweder G, Naalsund E, Lydersen S. Medical complications in a comprehensive stroke unit and an early supported discharge service. Stroke. 2008;39(2):414-20.

4. Ingeman A, Andersen G, Hundborg HH, Svendsen ML, Johnsen SP. In-hospital medical complications, length of stay, and mortality among stroke unit patients. Stroke. 2011;42(11):32148.

5. Lee SY, Chou CL, Hsu SP, Shih CC, Yeh CC, Hung CJ, Chen TL, Liao CC. Outcomes after stroke in patients with previous pressure ulcer: a Nationwide matched retrospective cohort study. Journal of Stroke and Cerebrovascular Diseases. 2016;25(1):220-7.

6. Nuru N, Zewdu F, Amsalu S, Mehretie Y. Knowledge and practice of nurses towards prevention of pressure ulcer and associated factors in Gondar University Hospital, Northwest Ethiopia. BMC nursing. 2015;14(1):34.

7. de M Engler TM, Dourado CC, Amâncio TG, Farage L, de Mello PA, Padula MP. Stroke: bowel dysfunction in patients admitted for rehabilitation. The open nursing journal. 2014;8:43.

8. Ashburn A, Hyndman D, Pickering R, Yardley L, Harris S. Predicting people with stroke at risk of falls. Age and ageing. 2008;37(3):270-6.

9. Armstrong M. Post discharge nursing care of stroke patients. American nurse today. February 2014; 9(2).

10. Rennke S, Ranji SR. Transitional care strategies from hospital to home: a review for the neurohospitalist. The Neurohospitalist. 2015;5(1):35-42.

11. Arts SE, Francke AL, Hutten JB. Liaison nursing for stroke patients: results of a Dutch evaluation study. J Adv Nurs. 2000;32(2):292-300.

12. Burton C, Gibbon B. Expanding the role of the stroke nurse: a pragmatic clinical trial. $J$ Adv Nurs. 2005;52(6):640-50.

13. Nasrabad RR. Introducing a new nursing care model for patients with chronic conditions. Electronic physician. 2017;9(2):3794.

14. Ribas ED, Bernardino E, Larocca LM, Poli Neto P, Aued GK, Silva CP. Nurse liaison: a strategy for counter-referral. Rev Bras Enferm. 2018;71:546-53. 
15. Tabanejad Z, Pazokian M, Ebadi A. The Effect of Liaison Nurse Service on Patient Outcomes after Discharging From ICU: a Randomized Controlled Trial. J Caring Sci. 2016;5(3):215.

16. Van Emden DM, Ros WJ, Berns MP. Transition of care: an evaluation of the role of the discharge liaison nurse in the Netherlands. $J$ Adv Nurs. 1999;30(5):1186-94.

17. Alberto L, Zotárez H, Cañete ÁA, Niklas JE, Enriquez JM, Gerónimo MR, del Carmen Martínez M, Chaboyer W. A description of the ICU liaison nurse role in Argentina. Intensive Crit Care Nurs. 2014;30(1):31-7.

18. 19). Tabanejad Z, Pazokian M, Ebadi A. A systematic review of the liaison nurse role on patient's outcomes after intensive care unit discharge. Int $J$ Community Based Nurs Midwifery. 2014;2(4):202.

19. Basami K, Mahdavi Z, Nikravan Mofrad M, Kohestani H, Baghcheghi N. Effects of disinfecting meatus and urinary catheter with $10 \%$ Povidone Iodine ointment on incidence rate of bacteriuria in hospitalized male patients in neurologic wards. Journal of Arak University of Medical Sciences. 2008 10;11(2):10-8. [Persian]

20. Azadmanesh Y, Azimian J, Jahani HH, Shahrokhi A, Naemian S. Comparing the effect of washing meatus urinary region with chlorhexidine and poidon iodine solutions on the appearance of bacteriuria in patients with fixed urinary catheter suffering from brain stroke (CVA). Modern Care Journal. 2013;10(4):241-8. [Persian]

21. Kazemnejad-Leili E, Rezaei S, Hosseini-Nejad M, Bakhshayesh-Eghbali B, Saberi A, Keshavarz P. The Applicability, Concurrent Validity and Internal Consistency Reliability of the Persian Version of the National Institutes of Health Stroke Scale (NIHSS): Evidences for Gender Differences. Caspian Journal of Neurological Sciences. 2016;2(1):18-28. [Persian]

22. Soozani A, Khosravi A, Pourheydari M, Montazeri A. Using Braden and Waterlow scales to predict pressure ulcer: a comparative study. Knowledge \& Health. 2011;5(4):43-8. [Persian]

23. Morse JM, Morse RM, Tylko SJ. Development of a scale to identify the fall-prone patient. Can J Aging. 1989;8(4):366-77.

24. Shali M, Joolaee S, Vaskooi KH, Bahrani N. Assessing the Patient Falls in Hospitals Affiliated to Tehran University of Medical Sciences. Iran Journal of Nursing. 2016;29(103):1-2. [Persian]

25. Kamali K, Glian Tehrani S, Banaei M, Jamshidi M, Mohseni S. A survey of prevalence of constipation and its associated factors in postmenopausal Women referred to health centers of Roudan-2015. The Iranian Journal of Obstetrics, Gynecology and Infertility. 2017;20(7):25-35. [Persian]

26. Lee J. Management of acute stroke complication. Journal of the Korean Medical Association. 2009;52(4):365-74.

27. Shaikh M. Fear of falling in patients with chronic stroke: differences of functional gait and balance measures according to the level of concern about falling. Journal of Rehabilitation Sciences \& Research. 2016;3(2):35-8. [Pdrsian]

28. Batchelor FA, Hill KD, Mackintosh SF, Said CM, Whitehead CH. Effects of a multifactorial falls prevention program for people with stroke returning home after rehabilitation: a randomized controlled trial. Arch Phys Med Rehabil. 2012;93(9):1648-55.

29. Teasell R, Foley N, Salter K, Bhogal S, Jutai J, Speechley M. Evidence-based review of stroke rehabilitation: executive summary. Top Stroke Rehabil. 2009;16(6):463-88.

30. Doshi VS, Say JH, Young SH, Doraisamy P. Complications in stroke patients: a study carried out at the Rehabilitation Medicine Service, Changi General Hospital. Singapore Med J. 2003;44(12):643-52.

31. Duncan PW, Zorowitz R, Bates B, Choi JY, Glasberg JJ, Graham GD, Katz RC, Lamberty K, Reker D. Management of adult stroke rehabilitation care: a clinical practice guideline. Stroke. 2005;36(9):e100-43.

32. Lo SH, Chang AM, Chau JP. Study protocol: a randomised controlled trial of a nurse-led community-based self-management programme for improving recovery among communityresiding stroke survivors. BMC Health Ser Res. 2016;16(1):387.

33. Sarfo FS, Treiber F, Jenkins C, Patel S, Gebregziabher M, Singh A, Sarfo-Kantanka O, Saulson R, Appiah L, Oparebea E, Ovbiagele B. Phone-based Intervention under Nurse Guidance after Stroke (PINGS): study protocol for a randomized controlled trial. Trials. 2016;17(1):436. 
